\title{
Free Vibration Analysis of a Rectangular Duct with Different Axial Boundary Conditions
}

\author{
Pruthviraj Namdeo Chavan and B. Venkatesham \\ Department of Mechanical Engineering, Indian Institute of Technology Hyderabad, Ordnance Factory Estate, \\ Yeddumailaram, 502205, A.P., India.
}

(Received 18 August 2012; revised 27 March 2014; accepted 7 May 2014)

\begin{abstract}
This paper describes the free vibration analysis of a rectangular duct by using the Rayleigh-Ritz method. Static beam functions are used as admissible functions in the Rayleigh-Ritz method. These basis functions are the static solutions of a point-supported beam under a series of sinusoidal loads. The unique advantage of using this method is that it allows for the consideration of different axial boundary conditions of a duct. Computational results are validated with existing literature data for a simply supported rectangular duct and the finite element method (FEM) for other axial boundary conditions. A validated analytical model is used for generating natural frequency data for different dimensions of rectangular ducts. Further curve fitting has been done for the generated data, and an empirical relation has been presented to calculate the first fundamental natural frequency for different material properties of ducts and different axial boundary conditions, which can be used for any dimensions of the duct within the specified range.
\end{abstract}

\section{INTRODUCTION}

Heating, Ventilation, and Air Conditioning (HVAC) systems extensively use ducts of different sizes and shapes that are connected in series or in parallel for air-handling purposes. The most prominent duct shapes are circular, rectangular, and elliptical. Noise generated from air handling units propagates in the axial and transverse directions of a duct. Noise radiated from ducts in the transverse direction is called breakout noise. Rectangular ducts have the highest breakout noise compared to a circular duct's cross-section due to lower stiffness. The breakout noise from these ducts has an impact at lower frequencies. The coupling of acoustic duct modes and structural duct modes plays a critical role in generating noise in the transverse direction. The first step in understanding structuralacoustic coupling is to calculate the natural frequencies and mode shapes of the structural components. The research interest in this paper is the free vibration analysis of rectangular ducts.

Different methods have been proposed in literature for free vibration analysis of polygonal ducts. S. Azimi et al. and G. Yamada et al. used the receptance method. ${ }^{1,2}$ H. P. Lee used the Rayleigh-Ritz method for calculating the natural frequencies and the mode shape of cylindrical polygonal ducts, in which sinusoidal functions are used as admissible functions. ${ }^{3}$ $\mathrm{T}$. Irea, et al. used the transfer matrix method for free vibration analysis of prismatic shells. ${ }^{4}$ Sai Jagan Mohan et al. used the Finite Element Method (FEM) to calculate the duct natural frequencies and the mode shape. ${ }^{5}$ They used group theoretical analysis to characterize duct modes. Existing literature results have only considered simply supported boundary conditions in the axial direction of the duct. According to current research, there is no work reported for the other axial boundary conditions. So in the present paper, the Rayleigh-Ritz method, which is capable of considering different axial boundary conditions, is used for calculating the natural frequencies of rectangular ducts.

A good amount of literature is available for the Rayleigh-
Ritz methods with different admissible functions. Selection of proper admissible functions provides variation in the RayleighRitz method. Admissible function varies based on applications like rectangular plates, rectangular plates with intermediate supports, etc. Zhou Ding used the Rayleigh-Ritz method for natural frequency analysis of rectangular plates with a set of static beam functions as admissible functions. ${ }^{6} \mathrm{D}$. Zhou et al. used a set of static beam functions for free vibration analysis of rectangular plates with intermediate supports. ${ }^{7}$ In the present paper, rectangular ducts are modelled as unfolded plates with rotational springs, and the creases are modelled as intermediate supports. The set of static beam functions are extended for the rectangular ducts. In the Rayleigh-Ritz method, validity and accuracy are entirely dependent on the choice of the admissible functions. ${ }^{8}$ These static beam functions are the static solutions of the point-supported beams under sinusoidal loads.

Calculated results from this method are validated with the data from the literature and with the FEM results. The validated analytical model is used to generate the engineering data for the rectangular ducts with different side ratios (height to width or width to height of the duct) and with different aspect ratios (perimeter of the duct cross section to length of the duct). These ratios are taken as four-step values $(0.25,0.5,0.75$, and 1). Further curve fitting has been done for generated engineering data results, and an empirical relation has been developed to calculate the first fundamental frequency. This empirical relation can be used to calculate the first fundamental frequency for any combination of an aspect ratio and a side ratio between a range of 0.25 to 1 with a prediction accuracy of $95 \%$.

Figure 1 shows the rectangular duct with dimensions of $L_{1}$, $L_{2}$, and $L_{3}$ in $X, Y$, and $Z$ directions, respectively. The reference coordinate system is also shown in Fig. 1.

\section{THE RAYLEIGH-RITZ METHOD}

The main advantage of this present Rayleigh-Ritz method with the static beam function as an admissible function is that 